\title{
Computational Simulation of the Random Depolymerization of Linear Polymers Obtained by Step-Polymerization of AB-type Monomers
}

\author{
Floralba López ${ }^{1}$, Geraldine Rangel ${ }^{1}$, Mayerlin Uzcátegui ${ }^{2}$ and Cristóbal Lárez Velásquez ${ }^{3, *}$ \\ ${ }^{1}$ Grupo de QUIFFIS, Departamento de Química, Facultad de Ciencias, Universidad de Los Andes, Mérida \\ 5101, Venezuela \\ ${ }^{2}$ SUMA, Departamento de Física, Facultad de Ciencias, Universidad de Los Andes, Mérida 5101, Venezuela \\ ${ }^{3}$ Grupo de Polímeros, Departamento de Química, Facultad de Ciencias, Universidad de Los Andes, Mérida \\ 5101, Venezuela
}

\begin{abstract}
This paper presents evaluation results of a computational algorithm developed to simulate the random depolymerization process of a linear polymer having an initial distribution of molecular weights similar to polymers obtained by polycondensation of AB-type monomers, i.e., Flory-Schultz or most probable distribution. Starting from fundamental definitions of this system, as the initial values of the degree of polymerization and chain number $\left(X_{\text {no }}\right.$ and $\mathrm{N}_{0}$, respectively) and the percentage of cleaved bonds (\%E), it is confirmed that our algorithm adequately describes the random depolymerization process. Results obtained during the computational simulation indicate that the algorithm properly predicts, among other things, that the inverse of the final polymerization degree $\left(1 / X_{n f}\right)$ increases linearly with the applied \%E while polydispersity after depolymerization $\left(D_{f}\right)$ decreases linearly when the latter parameter increases.
\end{abstract}

Keywords: Depolymerization computational simulation, Flory-Schultz distribution, polydispersity index, random scission.

\section{INTRODUCTION}

Changes in the molecular weight distribution (MWD) experienced by a polymer during a specific depolymerization process can provide accurate information about the reaction mechanism involved. On the one hand, the availability of modern techniques such as size exclusion chromatography (SEC) enables one to obtain more complete information on the degradative process in polymers because it is possible to know the MWD evolution, which obviously generates far more information than those provided by the sole evolution of averages of molecular weight (by number or weight) during such processes [1]. On the other hand, the emergence in recent years of computational tools available to many research groups, has allowed the development of algorithms that can simulate a wide range of situations involving polymer systems, which include depolymerization processes [2-4]. The combination of these two technological resources has allowed design strategies based primarily on building theoretical models that can be used for computational simulation of depolymerization processes, which can then be confirmed or not by SEC experimental studies.

*Address correspondence to this author at the Grupo de Polímeros, Departamento de Química, Facultad de Ciencias, Universidad de Los Andes, Mérida 5101, Venezuela; Tel: 582742401381; Fax: 582742401286;

E-mail: clarez@ula.ve
In this work, we present results obtained by means of a computational algorithm developed to simulate a random depolymerization process. In order to achieve reliable results, it was evaluated by simulating a random depolymerization of a quite simple polymeric system, whose relevant parameters, such as the number average degree of polymerization and dispersity $\left(X_{n}\right.$ and $D$, respectively) can be directly derived from their basic definitions as the polymerization extension, initial number of chains and bond scission percentage ( $p, N_{0}, \% E$, respectively). Thus, a linear polymeric system was chosen, with an initial MWD like those resulting from steppolymerization of A-B-type monomers, that is a FlorySchultz or most probable distribution.

\section{METHODOLOGY}

\subsection{Obtaining Samples with Well-Established Initial MWD}

The simple step-polymerization system considered can be defined according to:

$$
n A-B \rightarrow A-(B A)_{n-1}-B+(n-1) L
$$

where $A-B$ is the monomeric unit, $L$ is the low molecular weight species evolved in each step of the condensation reaction, and $\mathrm{n}$ is the number of monomeric units. 


\subsubsection{Mathematical Modeling Using the Flory- Schulz Distribution (FSD)}

From a given value for an initial number of monomer units $\left(\mathrm{UM}_{\mathrm{O}}\right)$ and a given extent of reaction $(p)$ the FSD is obtained by using the following equation [5]

$N_{x}=N p^{1-x}(1-p)=U M_{o} p^{1-x}(1-p)^{2}$

where

$\mathrm{N}_{\mathrm{x}}$ : number of polymer molecules with a degree of polymerization $\mathrm{x}$

$\mathrm{N}$ : total number of polymer molecules after polymerization reaction

$p=\left(U M_{O}-N\right) / U M_{O}$ : extent of the polymerization reaction

Parameters usually considered on polymer characterization are defined as:

$\mathrm{X}_{\mathrm{n}}=\Sigma \mathrm{xN}_{\mathrm{x}} / \Sigma \mathrm{N}_{\mathrm{x}}$ : number average degree of polymerization

$\mathrm{X}_{\mathrm{w}}=\Sigma \mathrm{x}^{2} \mathrm{~N}_{\mathrm{x}} / \Sigma \mathrm{x} \mathrm{N}_{\mathrm{x}}$ : weight average degree of polymerization

$D=X_{w} / X_{n}=\Sigma x^{2} N_{x} \Sigma N_{x} /\left(\Sigma x N_{x}\right)^{2}:$ polydispersity index or dispersity.

Additionally, $X_{n}$ can be obtained by Carothers equation:

$X_{n}=1 /(1-p)$

and the value of dispersity is given by:

$D=1+p$

\subsubsection{Discretization of the FSD}

MWD, obtained in the previous section, is discretized by converting each particular $N_{x}$ value to the closest integer. For the distribution thus discretized (DFSD), new values for the number average degree of polymerization $\left(X_{n d}\right)$, the total number of polymer molecules $\left(\mathrm{N}_{\mathrm{d}}\right)$, the total number of repeating units $\left(U M_{o d}\right)$ and the dispersity $\left(D_{d}\right)$ are obtained (by counting). It is noteworthy that differences in the number of chains before and after the discretization process can be observed, which also generates differences between $U_{0}$ and the total number of repeating units after discretization $\left(\mathrm{UM}_{\mathrm{od}}\right)$.

\subsubsection{Computational Simulation of Step- Polymerization}

Using pre-established values for $\mathrm{UM}_{0}$ and the extension of polymerization to be reached (denoted in this part as $p_{s}$ ) and a computational algorithm (Scheme 1) that simulates the random bonding among molecular species of any size generated during the polymerization process (equal reactivity principle), a simulated molecular weight distribution was obtained (denoted as SIMD-A, with the A value indicating the number of experiments averaged).

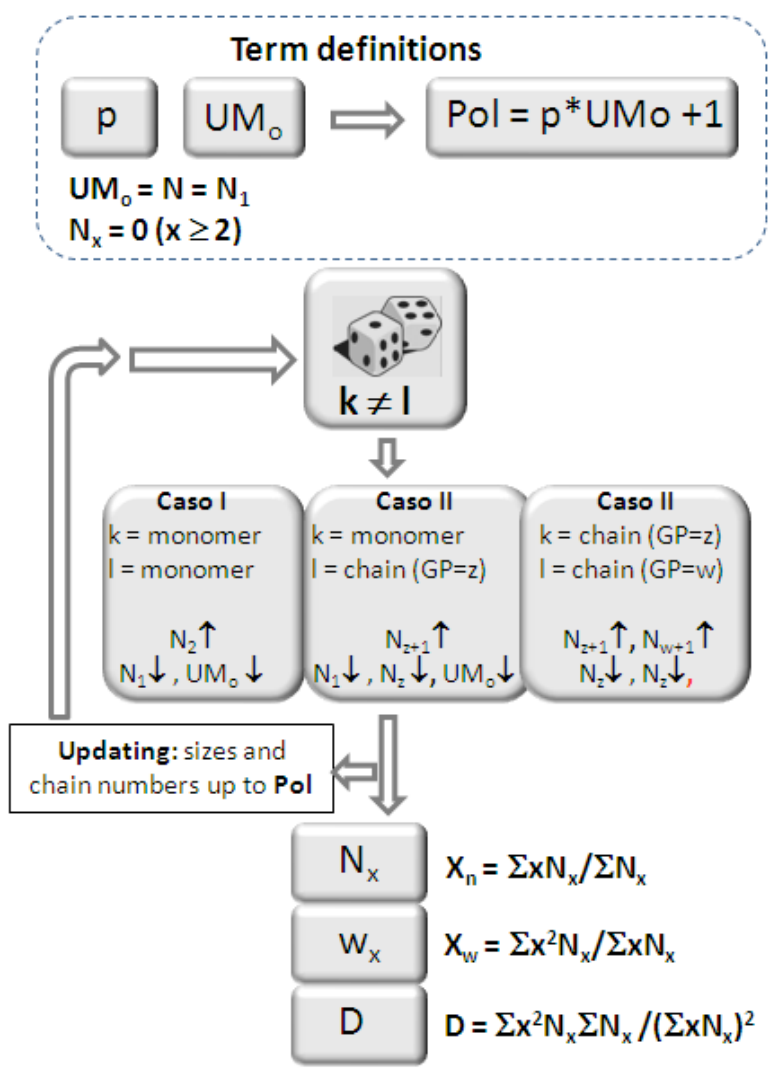

Scheme 1:

\subsection{Computer Simulation of Depolymerization Process}

Samples with initial MWD previously obtained by DFSD and SIMD, are used in the depolymerization simulation for well defined initial values for $\mathrm{N}$ and $\mathrm{p}$, using just the percentage of bonds to be cleaved (\%E), occurring for such random breakdown only between repetitive units forming part of all chains present in the sample (Scheme 2). It is important to mention that when DFSD is employed as the input data, the depolymerization process simulation is performed only once because these data are initially obtained by a function generating the most probable values for each 
value of $N_{x}$. Meanwhile, when using SIMD-A as the input data, it is necessary to perform many simulation experiments to obtain an average value because the realization of a single experiment generates just one of many possible values for the studied parameters.

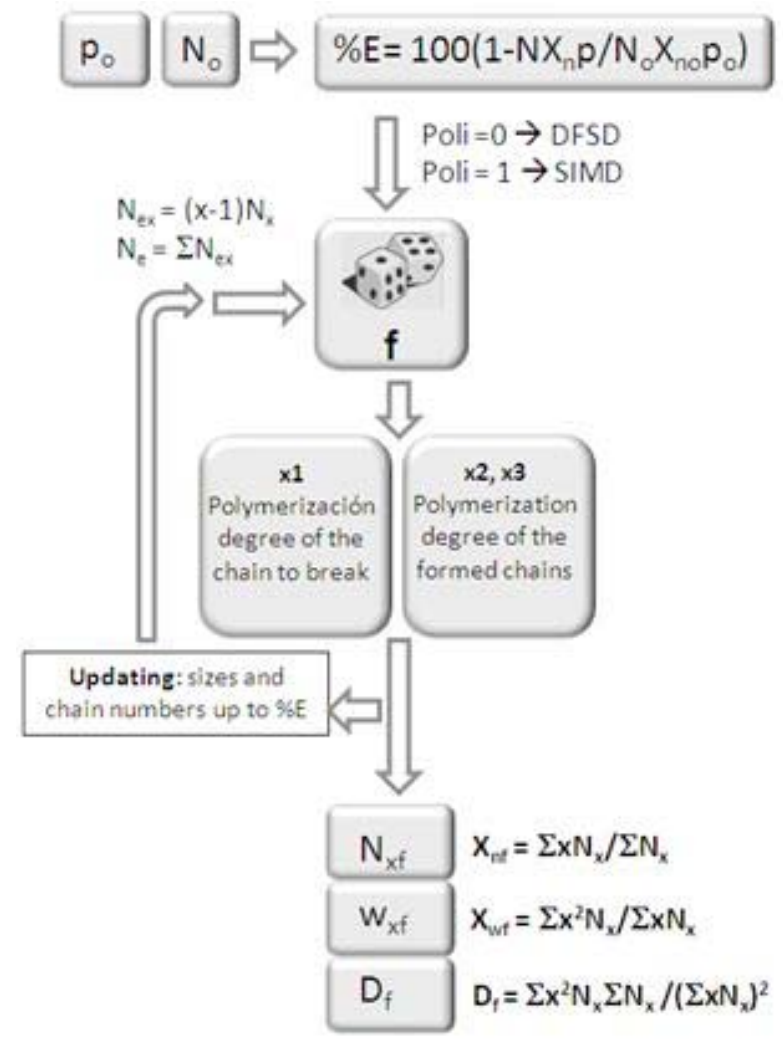

Scheme 2:

\subsubsection{Initial Samples with DFSD}

After introducing the input data $\left(\mathrm{UM}_{0}, \mathrm{p}, \% \mathrm{E}\right)$, the computational algorithm performs the following process:

a) Discretization of the FSD generating, by counting, values for $X_{n d}, X_{w d}, N_{d}, U M_{o d}$ and $D_{d}$.
The subscript $d$ refers to values obtained after discretization of the FSD.

b) Simulation of the random depolymerization process generating, by counting, the final values (after cleavage) for $X_{n d f}, X_{w d f}, N_{d f}, U M_{\text {df }}$ and $D_{d}$. The subscript $f$ refers to values obtained after the depolymerization process simulation.

\subsubsection{Initial Samples with SIMD-A}

Following the introduction of the input data $\left(\mathrm{UM}_{\mathrm{o}}, \mathrm{p}_{\mathrm{s}}\right.$ and $\% \mathrm{E}$ ), the computational algorithm performs the following process:

a) Simulation of the polymerization to complete the reaction up to the desired $p_{s}$ value generating, by counting, values for $X_{n s}, X_{w s}, N_{s}, U M_{o s}$ and $D_{s}$. The subscript $s$ refers to values obtained after the polymerization process simulation.

b) Simulation of the random depolymerization process generating, by counting, the final values (after cleavage) for $\mathrm{X}_{\mathrm{nsf}}, \mathrm{X}_{\mathrm{wsf}}, \mathrm{N}_{\mathrm{sf}}, \mathrm{UM}_{\mathrm{osf}}$ and $\mathrm{D}_{\mathrm{sf}}$. The subscript $f$ refers to the values obtained after depolymerization process simulation.

\section{RESULTS AND DISCUSSION}

\subsection{Comparison of MWDs Obtained for the Initial Sample}

Since the computational algorithm for simulating the depolymerization process was developed to cleave samples containing an integer number of chains, of any size, with integer numbers of repetitive units, an initial attempt was made using as input data the DFSD. Table 1 shows the values of $X_{n}$ obtained from each one of the MWD generated, for the set of values $p$ and $N$ evaluated. It can be clearly appreciated that values of

Table 1: Comparison of the Values $X_{n d}$ and $X_{n s}$ with the Values of $X_{n}$ Obtained from the FSD for Different $p$ and $N$ Values

\begin{tabular}{|c|c|c|c|c|c|c|c|c|c|c|c|}
\hline \multirow[b]{2}{*}{$\mathbf{p}$} & \multicolumn{5}{|c|}{$X_{n d}$} & \multirow{2}{*}{$\frac{X_{n}}{1 /(1-p)}$} & \multicolumn{5}{|c|}{$X_{n s}$} \\
\hline & $1 \times 10^{3}$ & $1 \times 10^{4}$ & $\underset{1 \times 10^{5}}{N}$ & $5 \times 10^{5}$ & $1 \times 10^{6}$ & & $1 \times 10^{3}$ & $1 \times 10^{4}$ & $\underset{1 \times 10^{5}}{N}$ & $5 \times 10^{5}$ & $1 \times 10^{6}$ \\
\hline 0.90000 & 9.824 & 9.974 & 9.998 & 9.999 & 9.999 & 10 & 10.000 & 10.000 & 10.000 & 10.000 & 10.000 \\
\hline 0.95000 & 19.268 & 19.899 & 19.987 & 19.997 & 19.999 & 20 & 19.980 & 19.998 & 19.999 & 19.999 & 19.999 \\
\hline 0.99000 & 87.563 & 98.017 & 99.731 & 99.936 & 99.966 & 100 & 99.900 & 99.990 & 99.999 & 99.999 & 99.999 \\
\hline 0.99500 & 158.246 & 192.865 & 198.968 & 199.745 & 199.862 & 200 & 199.800 & 199.980 & 199.998 & 199.999 & 199.999 \\
\hline 0.99875 & 367.000 & 715.870 & 786.624 & 796.629 & 798.161 & 800 & 800.000 & 800.000 & 800.000 & 800.000 & 800.000 \\
\hline 0.99900 & 347.500 & 874.613 & 979.855 & 994.862 & 997.207 & 1000 & 999.001 & 999.900 & 999.990 & 999.998 & 999.999 \\
\hline
\end{tabular}




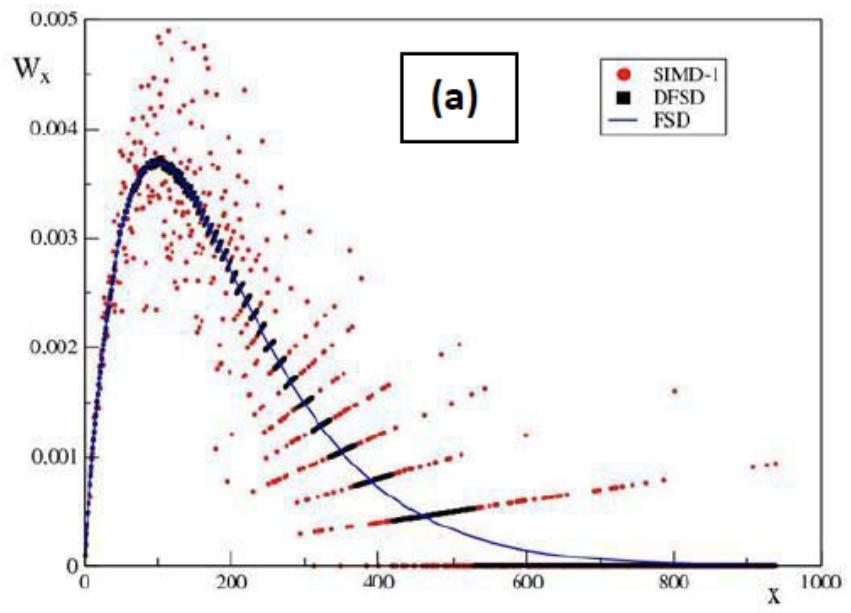

Figure 1: Comparison between (a) FSD, DFSD and SIMD-1; (b) $1 \times 10^{4}$.

$X_{\text {nd }}$ (from DFSD) differ significantly from values of $X_{n}$ (from FSD) when small values of $\mathrm{N}$ and high values of $p$ are considered. Values of $X_{n d}$ which differ by more than $1 \%$ of the $X_{n}$ value have been highlighted.

Due to the above results, a computational algorithm was developed to simulate the polymerization of this type of system, which considered the principle of equal reactivity as a fundamental premise for the construction of the chains. As can be observed in Table 1, the $X_{n s}$ values obtained by means of this algorithm (values generated by the algorithm simulating the polymerization process) appear to produce better values than the discretization of the FSD since $X_{n s}$ values are closer to the values of $X_{n}$ than those obtained for $X_{n d}$. A comparison is performed with $X_{n}$ because this is a generally accepted parameter to characterize these systems and since it is very easy to compute using the Carothers equation (Equation 5).

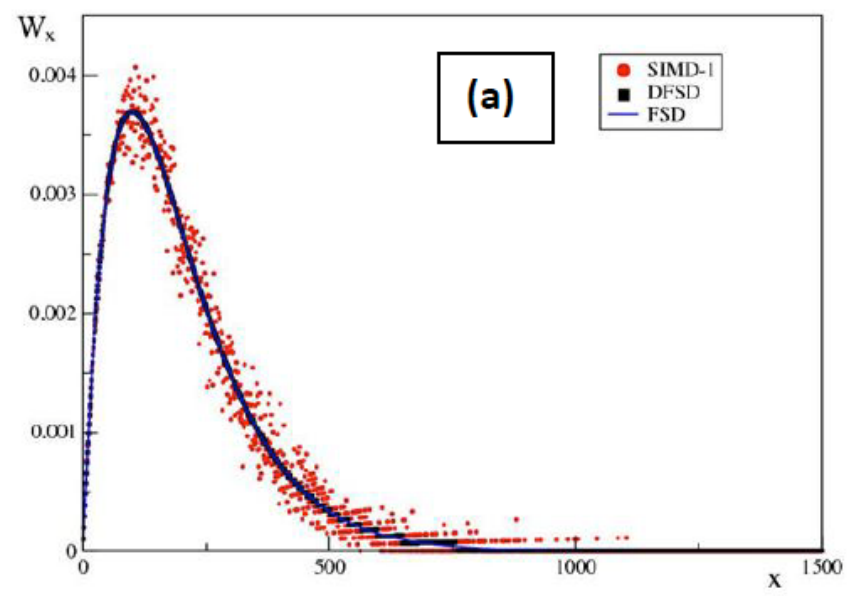

Figure 2: Comparison between (a) FSD, DFSD and SIMD-1; (b) $1 \times 10^{5}$.

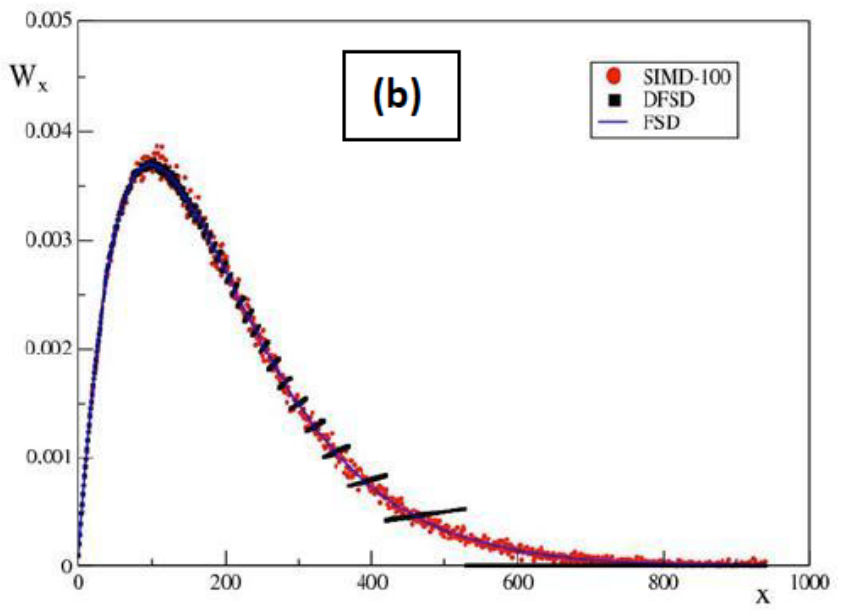

(b) DFS, DFSD and SIMD-100. In both cases $p=0.99$ and $\mathrm{N}=$

Nevertheless, when the resulting distributions of both processes (DFSD and SIMD-1) are compared with FSD it can be appreciated that DSIM-1 produces significant fluctuations in the $\mathrm{w}_{\mathrm{x}}$ values (Figure 1a) although it also should be noted that such fluctuations occur around the expected $w_{x}$ values, i.e., in the neighborhood of $w_{x}$ predicted by the FSD. Such inconvenience can be largely overcome by averaging the $w_{x}$ values from an appropriate number of simulation experiments, as it is demonstrated in Figure $\mathbf{1 b}$, which shows clearly how these fluctuations decrease when results from 100 simulation experiments are averaged (SIMD-100) for the same initial values of $p=0.99$ and $\mathrm{N}=1 \times 10^{4}$ employed to obtain the Figure $1 \mathrm{a}$.

Similarly, fluctuations in the values of $w_{x}$ also decrease when considering higher values of $\mathrm{N}$, as shown in Figure $\mathbf{2 a}$ and $\mathbf{2 b}$, which compares SIMD-1 and SIMD-100, respectively, with the DFS and DFSD, for values of $p=0.99$ and $\mathrm{N}=1 \times 10^{5}$.

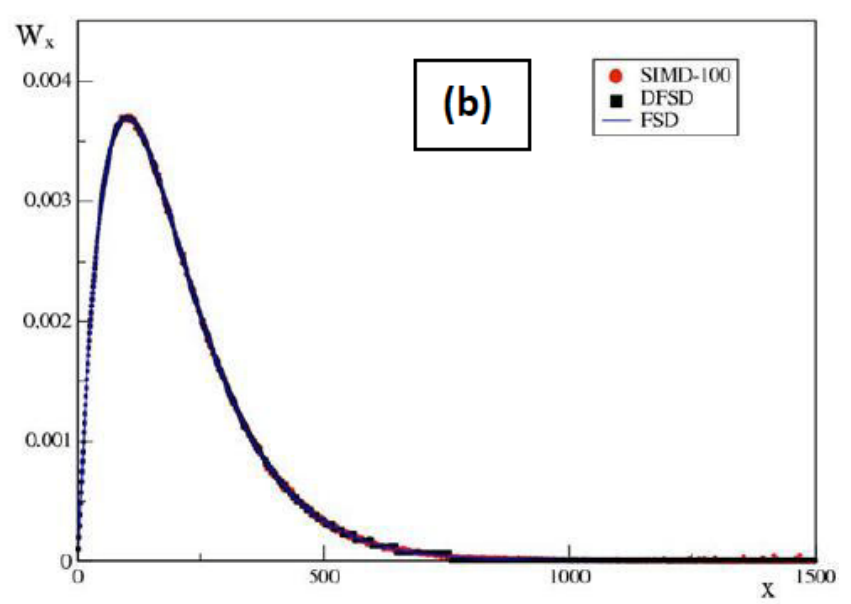


Table 2: Parameters Evaluated During the Computational Simulation of Depolymerization Processes

\begin{tabular}{|c|c|c|c|}
\hline Condition & \%E & $\mathbf{X}_{\text {no }}$ & $\mathbf{N}_{\circ}$ \\
\hline \hline I & Variable & Constant & Constant \\
\hline II & Constant & Variable & Constant \\
\hline III & Constant & Constant & Variable \\
\hline
\end{tabular}

Previous results allow concluding that the number of chains present in the initial sample of the polymer is an important parameter to consider in the simulation of the depolymerization reactions. In this regard, all depolymerization simulations were performed using samples with initial values of $p=0.99$ and $N=1 \times 10^{5}$, considering that these values allow computational simulation to run in reasonable times.

\subsection{Computational Simulation of the Depolymerization Reactions}

We studied the effects of the following parameters in the initial sample: number average degree of polymerization $\left(\mathrm{X}_{\mathrm{no}}\right)$, cleavage percentage applied $(\% \mathrm{E})$ and the number of chains $\left(\mathrm{N}_{\mathrm{o}}\right)$, on final values of the system such as $1 / X_{n f}$ and $D_{f}$. Table 2 provides a summary of the conditions under which the computational simulation studies of depolymerization were conducted.

\subsubsection{Condition I}

Figure 3 shows the resulting MWDs from the depolymerization computational simulation of an initial sample with values of $X_{n o}=100$ and $N=1 \times 10^{5}$, which was evaluated for different \%E values. It can be clearly observed that all final MWDs are displaced toward lower values of $x$ and also that they become more narrower. Table 3 shows the $X_{\text {nse }}$ and $D_{\text {se }}$ values obtained for the different \%E evaluated.

One of the most commonly used criteria to conclude that a depolymerization reaction occurs by a random scission mechanism is the existence of a linear relationship between $1 / \mathrm{X}_{\mathrm{nf}}$ and the reaction time (t), as it was proposed by Jellinek [6] through an equation similar to:

$1 / X_{n}=k t+1 / X_{n o}$

where $\mathrm{k}$ is the rate constant of the depolymerization process and $X_{n o}$ is de number average polymerization degree of the initial sample.

Some researchers have used this criterion to state that their experimental degradation studies take place through a mechanism of scission at random points of the chains. Thus, in their experimental studies on the microbial hydrolytic degradation of the copolyesters poly(3-hydroxy-butyrate-co-3-hydroxy-valerate) and poly(3-hidroxi-butyrate-co-4-hydroxy-butyrate) Doi et al. [7] obtained a linear relationship for $1 / X_{n} v s$. t. These

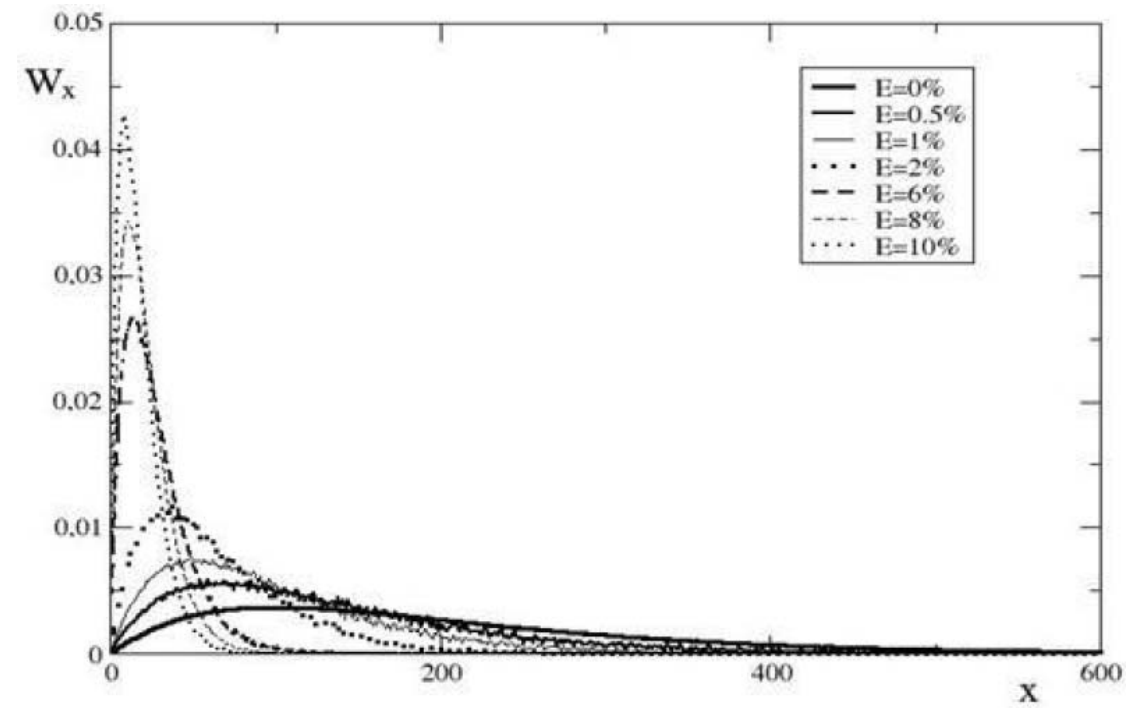

Figure 3: Final MWDs obtained after the simulation of depolymerization from an initial sample with $\mathrm{X}_{\mathrm{no}}=100$ and $\mathrm{N}=1 \times 10^{5}$ for the different \%E shown. All curves were obtained by averaging 100 simulations runs. 
Table 3: Values of $D_{s f}$ and $X_{n s f}$ after of the Depolymerization Computational Simulation of an Initial Sample with $X_{n o}=100$ and $\mathrm{N}_{\mathrm{o}}=1 \times 10^{5}$. All Values were Obtained by Averaging 100 Simulation Runs

\begin{tabular}{|c|c|c|c|}
\hline$\% \mathrm{E}$ & $\mathbf{X}_{\mathrm{nsf}}$ & $\mathbf{1 / \mathbf { X } _ { \text { nsf } }}$ & $\mathbf{D}_{\text {sf }}$ (SIMD-100) \\
\hline \hline 0.0 & 100.00 & 0.010 & 1.990 \\
\hline 0.5 & 59.22 & 0.017 & 1.983 \\
\hline 1.0 & 47.88 & 0.021 & 1.979 \\
\hline 2.0 & 32.62 & 0.031 & 1.969 \\
\hline 4.0 & 19.61 & 0.051 & 1.949 \\
\hline 6.0 & 13.85 & 0.072 & 1.928 \\
\hline 8.0 & 10.60 & 0.094 & 1.906 \\
\hline
\end{tabular}

results are similar to those obtained by Lauzier et al. [8] during the hydrolytic degradation of the poly ( $\beta$ hydroxy-butyrate) and Kunioka and Doi [9] from the thermal hydrolysis of the copolyesters poly(3-hydroxybutyrate-co-3-hydroxy-valerate) and poly(3-hidroxibutyrato-co-4-hydroxy-butyrate).

Simulations performed in the present study do not explicitly consider the kinetic aspect of the depolymerization reaction; however, the plot of $1 / \mathrm{X}_{\mathrm{nf}} v s$. $\% E$ showed a clear linear relationship (Figure 4).

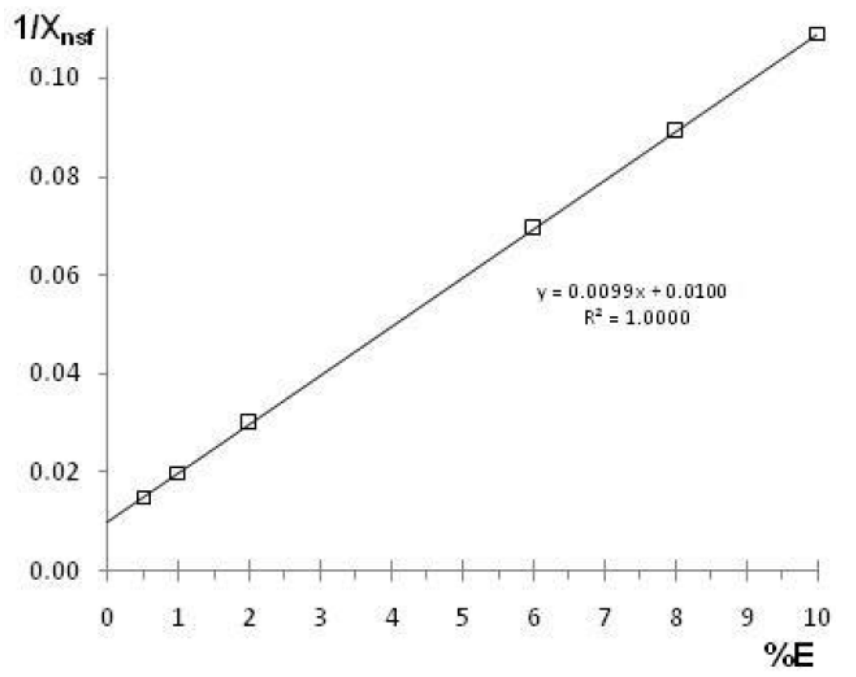

Figure 4: Plot of $1 / \mathrm{X}_{\text {nsf }}$ vs. \%E obtained by using results from depolymerization computational simulations of an initial sample with $\mathrm{X}_{\mathrm{no}}=100$ and $\mathrm{N}=1 \times 10^{5}$.

The straight line obtained from the plot $1 / \mathrm{X}_{\text {nst vs. }} \% \mathrm{E}$ may be entirely explained by considering the definition for \%E obtained from the basic parameters of the system, which produce the following relationship:

$\% E=\left(n_{e o}-n_{e}\right) \cdot 100 / n_{e o}=100\left(1-n_{e} / n_{e o}\right)$

where:
$\mathrm{n}_{\mathrm{eo}}=\mathrm{N}_{\mathrm{o}} \mathrm{X}_{\mathrm{no}}-\mathrm{N}_{\mathrm{o}}=\mathrm{N}_{\mathrm{o}}\left(\mathrm{X}_{\mathrm{no}}-1\right)=\mathrm{N}_{\mathrm{o}} \mathrm{p}_{\mathrm{o}} \mathrm{X}_{\mathrm{no}}$ : total number of bonds in the initial sample

$\mathrm{n}_{\mathrm{e}}=\mathrm{N} \mathrm{X}_{\mathrm{n}}-\mathrm{N}=\mathrm{N}\left(\mathrm{X}_{\mathrm{n}}-1\right)=\mathrm{Np} \mathrm{X}_{\mathrm{n}}$ : total number of bonds in the sample after depolymerization simulation

By substituting $n_{e o}$ and $n_{e}$ into equation 8 it becomes:

$\% E=100\left(1-N X_{n} p / N_{0} X_{n o} p_{0}\right)$

Because $\mathrm{N}_{0} \mathrm{X}_{\mathrm{no}}=\mathrm{N} \mathrm{X}_{\mathrm{n}}$, equation 9 simplifies to:

$\% E=100\left(1-p / p_{o}\right)$

By reordering it:

$$
\begin{aligned}
& -p / p_{o}=\% E / 100-1 \\
& -p=p_{o} \% E / 100-p_{o}
\end{aligned}
$$

By adding 1 to each side of the preceding equation it becomes:

$$
1-p=p_{0} \% E / 100+1-p_{0}
$$

Finally, substituting $1-p$ and $1-p_{o}$ by $1 / X_{n}$ and $1 / X_{n o}$, respectively, in the preceding equation, the required relationship is derived:

$1 / X_{n}=p_{0} \% E / 100+1 / X_{n o}$

According to equation 11 a plot of $1 / \mathrm{X}_{\mathrm{nst}}$ vs. \%E ( $\mathrm{p}_{\mathrm{o}}$ constant) should produce a straight line with slope $m=$ $p_{o} / 100$ and intercept $b=1 / X_{n o}$, as is perfectly observed in Figure 4. Values for $p_{0}$ and $X_{n o}$ obtained from $m$ and $b$ are presented in the Table 4.

Equation 11 also allows generating a direct relationship between \%E and $t$ by substituting $1 / X_{n}$ according to equation 7 : 
Table 4: Comparison of the Sample Initial Values $\left(p_{o}=0.99, X_{n o}=100, D_{0}=1.99\right)$ with those Computed by Using $m$ and b Values Obtained from Plots of $1 / X_{\text {nsf }}$ vs. $\% E$ and $D_{\text {sf }}$ vs. $\% E$

\begin{tabular}{|c|c|c|c|}
\hline \multirow{2}{*}{ Plot } & \multicolumn{3}{|c|}{ Initial sample values obtained from the plots* } \\
\hline & po & $X_{\text {no }}$ & $\mathbf{D}_{0}$ \\
\hline $1 / X_{n s t} v s . \% E(m=0.00990 ; b=0.0100)$ & $100 \mathrm{~m}=0.99$ & $1 / b=100$ & $1+p_{o}=1.99$ \\
\hline $\mathrm{D}_{\mathrm{sf}} v s . \% \mathrm{E}(\mathrm{m}=-0.00987 ; \mathrm{b}=1.98978)$ & $\begin{aligned}-100 m & =0.987 \\
D_{0}-1 & =0.990\end{aligned}$ & $\begin{array}{l}1 /\left(1-p_{\circ}\right)=77 \\
1 /\left(1-p_{\circ}\right)=98\end{array}$ & $\begin{array}{c}1+p_{o}=1.987 \\
b=1.990\end{array}$ \\
\hline
\end{tabular}

*Bold values were computed directly by using $m$ and $b$ values obtained from each straight line. Values in regular type were computed using the corresponding value shown in the respective equation. which is taken from its own row in the table.

$\% E=100 k t / p_{\circ}$

Thus, a plot of \%E vs.t ( $p_{0}$ constant) should produce a straight line from whose slope $m=100 \mathrm{k} / \mathrm{p}_{\mathrm{o}}$ the value of the rate constant $\mathrm{k}$ of the depolymerization process can be computed.

Furthermore, it is possible to establish a relationship between the final polydispersity of the system as a function of \%E applied, by considerations similar to those shown in the previous section, which leads to the following equation for $D_{f}$ :

$D_{f}=D_{0}-p_{\circ} \% E / 100$

Figure 5 shows the graph obtained for $\mathrm{D}_{\mathrm{sf}} v s$. \%E using the values generated by the computational simulation of the depolymerization process. It may be appreciated that an excellent straight line is obtained which produces values for $p_{0}$ and $D_{0}$ consistent with values obtained above (Table 4).

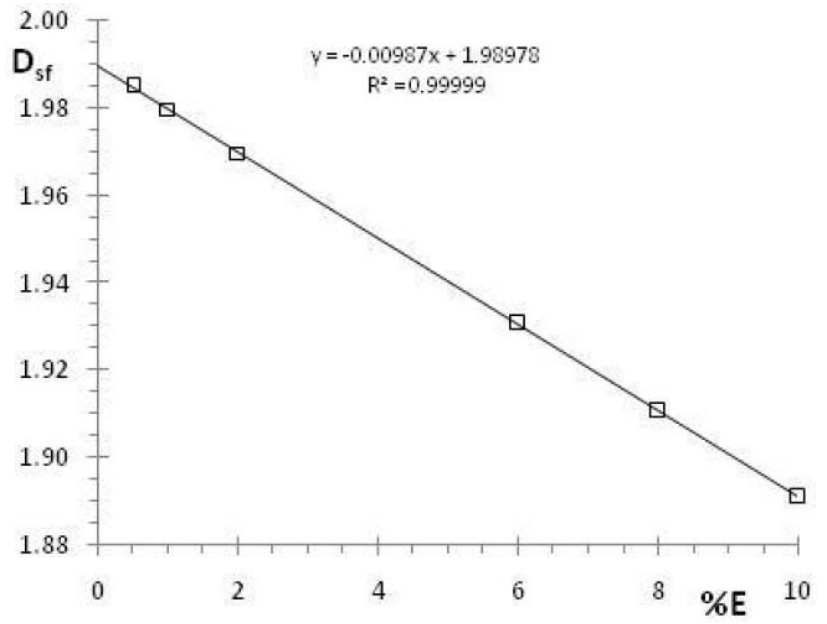

Figure 5: Plot of $D_{s f}$ vs. \%E using results from depolymerization computational simulations of an initial sample with $X_{n o}=100$ and $\mathrm{N}=1 \times 10^{5}$.

\subsubsection{Condition 2}

Figure 6 shows results obtained by plotting the values of $D_{f}$ coming from the depolymerization simulations for samples with different values of $X_{\text {no }}$ and maintaining a \%E constant. As it may be appreciated, all obtained straight lines tend to a maximum value for $D_{f}$ when $1 / X_{n o}$ tends to zero, i.e., $X_{n o} \rightarrow \infty$ (hereinafter $\left.D_{\infty}\right)$. Likewise, and according to their associated linear equations, all of them satisfy the following relationship:

$D_{s f}=b+m / X_{n o}=D_{\infty}+\left(1-D_{\infty}\right) / X_{n o}$

The dotted line is obtained for $D_{f}$ values expected for each of the initial samples (\%E =0); in this case the value of $D \rightarrow 2$ when $1 / X_{n o} \rightarrow 0$ because $X_{n o} \rightarrow \infty$.

In order to find an equation similar to equation 13 based on fundamental parameters of the system, with the purpose of predicting the behavior of $D_{\text {se }}$ as function of $1 / X_{n o}$, equation 11 can be rearranged as follows:

$p=p_{o}-p_{o} \% E / 100$

$1+\mathrm{p}=1+\mathrm{p}_{\mathrm{o}}-\mathrm{p}_{\mathrm{o}} \% \mathrm{E} / 100=1+\mathrm{p}_{\mathrm{o}}(1-\% \mathrm{E} / 100)=1+(1$ $\left.-1 / X_{n o}\right)(1-\% E / 100)$

$D_{s f}=(2-\% E / 100)+(\% E / 100-1) / X_{n o}$

Equation 14 is similar to the equation obtained using the data coming from depolymerization simulation; from it, one can obtain the values for:

$$
D_{\infty}=2-\% E / 100
$$

therefore the slope will be:

$$
m=\left(1-D_{\infty}\right)=\% E / 100-1
$$

The observed linear behavior of the graphs $D_{\mathrm{sf}} v s$. $1 / X_{n o}$ obtained by using the values of $D_{\text {se }}$ from the depolymerization computational simulation, maintaining constant values for \%E, fits perfectly with expectations from fundamental definitions, validating also by this route the proper functioning of the computational algorithm created for this simulation. 


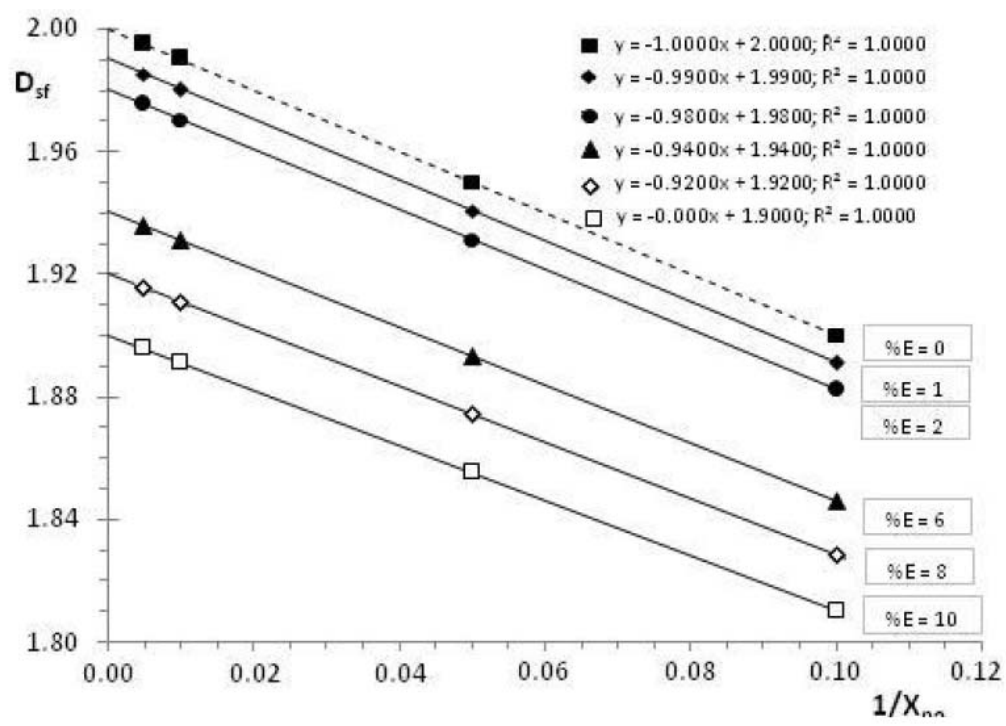

Figure 6: Plots for $D_{s f} v s .1 / X_{n o}$ using results from depolymerization computational simulations of an initial sample with $N=1 \times 10^{5}$ and maintaining constant the \%E values shown.

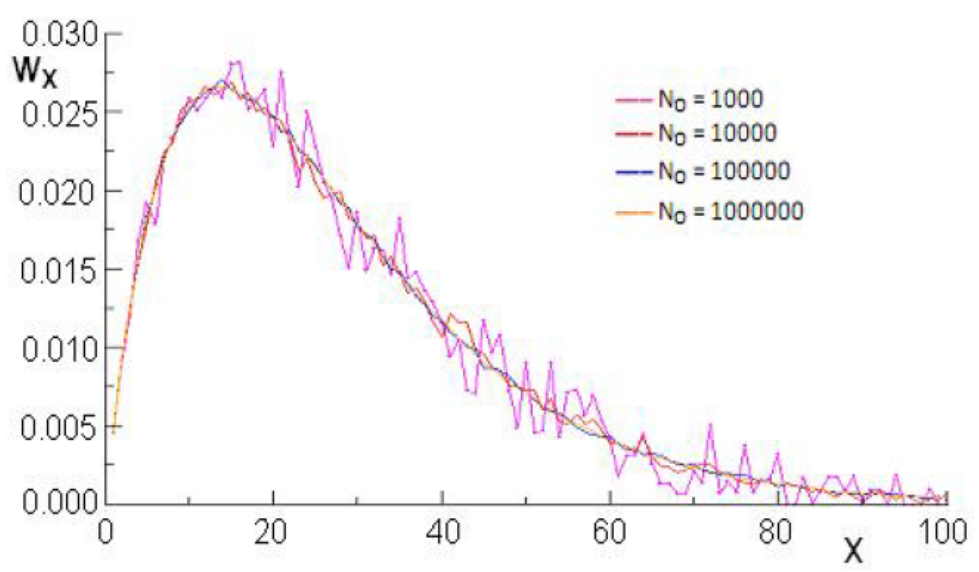

Figure 7: MWDs obtained after of depolymerization computational simulation at $\% \mathrm{E}=6 \%$ of an initial sample with $\mathrm{X}_{\mathrm{no}}=100$ for the $\mathrm{N}_{0}$ values shown.

\subsubsection{Condition 3}

Figure 7 shows the MWDs obtained after the depolymerization simulation at different \%E (constant) of an initial sample with $X_{n o}=100$, and varying the initial number of chains. MWDs are very similar to large values of $N_{0}$, while $w_{x}$ values show oscillations, that are more significant, for lower values of $\mathrm{N}_{0}$.

Because the MWDs remained without an apparent displacement with increasing $N_{0}$, it is expected that dispersity will not be affected by varying $N_{0}$, at least for high values of $N_{0}$, where the $w_{x}$ values show less oscillations. The plot from $D_{s f} v s . N_{\circ}$ in Figure 8 confirms this, showing that $D_{\text {ef }}$ remains practically constant and does not depend on $\mathrm{N}_{0}$, although it is important to notice that it shows fluctuations for low $\mathrm{N}_{\circ}$ values (not shown), which can perhaps be explained since the MWDs fail to be well defined for low $N_{\circ}$ values.
The constant values of $D_{s f}$ for sufficiently high values of $\mathrm{N}_{0}$ can also be explained by equations derived from the fundamental definitions of the system. Thus, because $1 / X_{n o}=N_{o} / X_{n} N=$ constant, equation 12 can be rearranged as follows:

$D_{s f}=D_{0}-\left(1-1 / X_{n o}\right) \% E / 100=$

$D_{s f}=D_{0}-\left(1-N_{o} / X_{n} N\right) \% E / 100=$

$D_{s f}=D_{o}-\% E / 100-\left(\% E / 100 X_{n} N\right) N_{o}$

The value of $D_{\text {sf }}$ predicted by this equation appears to have a linear dependence on $\mathrm{N}_{0}$; however, as $\mathrm{N}_{\mathrm{o}} / \mathrm{N}_{\mathrm{n}} \mathrm{N}$ remains constant while $\mathrm{X}_{\text {no }}$ stays constant, the equation manages to explain values obtained for $D_{\mathrm{sf}}$, which are constant and independent of $\mathrm{N}_{0}$. Another important point that, can be noted from equation 15 , is that the higher $\mathrm{X}_{\text {no }}$ and lower the \%E applied, the value of $D_{s f}$ will approach $D_{0}-\% E / 100$. 


\section{CONCLUSIONS}

The discretization process applied to the FlorySchulz distribution generates MWDs having discrepan-cies between the initial $U_{0}$ value and final number of repetitive units, which are particularly pronounced for low $\mathrm{N}_{\circ}$ and high $\mathrm{p}$ values.

The computational algorithm developed to simulate the step-polymerization of AB-type monomers allows obtaining MWDs without disagreements between $\mathrm{UM}_{0}$ and the final numbers of repetitive units, although the $w_{x}$ values show very large fluctuations. However, this problem can be minimized by averaging a sufficient number of simulation runs.

The computational algorithm developed to simulate the depolymerization by a random scission mechanism of a linear polymer obtained by step polymerization of AB-type monomers produces results consistent with those expected from the basic definitions of such systems, i.e. linear relationships for $1 / X_{n f}$ and $D_{f}$ as these are plotted as a function of $\% E$, maintaining $X_{n o}$ and $N_{o}$ constants; a linear relationship for the $D_{f}$ vs. $1 / X_{\text {no }}$ plot ( $\% E$ and $N_{0}$ constants); and $D_{f}$ was shown to remain independent of $\mathrm{N}_{\circ}$ for high values of $\mathrm{N}_{0}$, with \%E and $\mathrm{X}_{\mathrm{no}}$ constants. Thus, this algorithm may be considered ready for dealing with depolymerization simulation studies of linear polymeric systems similar to the one used in this study as a model, provided that their experimental MWDs can be adequately either simulated or discretized. Likewise, it should be possible to successfully simulate the random depolymerization of linear systems with different MWDs than those used in this study (wider or narrower), provided that their experimental MWDs can be appropriately discretized. Finally, the algorithm should be easily adjusted for simulating kinetic studies of random depolymerization reactions through the establishment of the constant of proportionality between $\% \mathrm{E}$ and the reaction time, i.e. the rate constant of the depolymerization process.

\section{ACKNOWLEDGEMENTS}

This work was supported by CDCHT-ULA through Project No. ADG-C09-95.

\section{REFERENCES}

[1] ledema $P$, Willems C, Vliet G, Bunge W, Mutsers S, Hoefsloot $H$. Using molecular weight distributions to determine the kinetics of peroxide-induced degradation of polypropylene. Chem Eng Sci 2001; 56: 3659-69. http://dx.doi.org/10.1016/S0009-2509(01)00054-9

[2] Bose S, Git Y. Mathematical Modelling and Computer Simulation of Linear Polymer Degradation: Simple Scissions. Macromol. Theory Simul 2004; 13: 453-73. http://dx.doi.org/10.1002/mats.200300036

[3] Garcia J, Koelling K, Summers. Computational prediction of PVC degradation during injection molding in a rectangular channel. J Polym Eng Sci 2004; 44: 1295-12. http://dx.doi.org/10.1002/pen.20125

[4] Bystritskaya E, Karpukhin O, Kutsenova A. Monte Carlo Simulation of Linear Polymer Thermal Depolymerization under Isothermal and Dynamic Modes. Int J Polym Sci vol. 2011, Article ID 849370, 6 pages, 2011. http://dx.doi.org/doi:10.1155/2011/849370

[5] Odian G. Principles of Polymerization. $4^{\text {th }}$ ed. Ed.: John Wiley \& Sons, Inc., Hoboken, New Jersey USA 2004.

[6] Jellinek $\mathrm{H}$. Some aspects of polymer degradation process. In: Effects of Polymer Degradation on Flow Properties of Fluids and Lubricants, pp. 3-13, 1965. ASTM Committee D-2 on Petroleum Products and Lubricants.

[7] Doi Y, Kanesawa Y, Kunioka M, Saito T. Biodegradation of microbial copolyesters: Poly(3-hydroxy-butyrate-co-3hydroxyvalerate) and Poly(3-hydroxybutyrate-co-4-hydroxylbutyrate). Macromolecules 1990; 23: 26-31. http://dx.doi.org/10.1021/ma00203a006

[8] Lauzier C, Revol J, Debzi E, Marchessault R. Hydrolytic degradation of isolated poly( $\beta$-hydroxy-butyrate) granules. Polymer 1994; 35(19): 4156-62.

http://dx.doi.org/10.1016/0032-3861(94)90590-8

[9] Kunioka Y, Doi Y. Thermal degradation of microbial copolyesters: poly(3-hydroxybutyrate-co-3-hydroxy-valerate) and poly(3-hydroxybutyrate-co-4-hydroxybutyrate). Macromolecules 1990; 23: 1933-36.

http://dx.doi.org/10.1021/ma00209a009 